\title{
CREACIÓN Y EVALUACIÓN DE PLANTILLAS PARA TRABAJOS FIN DE GRADO COMO BUENA PRÁCTICA DOCENTE
}

\section{CREATION AND EVALUATION OF FINAL DEGREE PROJECT TEMPLATES AS A GOOD TEACHING PRACTICE}

\author{
José Manuel Redondo López ${ }^{1}$
}

Fecha de recepción: 15/01/2020; Fecha de revisión: 18/03/2020; Fecha de aceptación: 25/06/20

Cómo citar este artículo:

Redondo, J. (2020). Creación y evaluación de plantillas para trabajos de fin de grado como buena práctica docente. Revista de Innovación y Buenas Prácticas Docentes, 9(2), 17-35.

Autor de Correspondencia: redondojose@uniovi.es

Resumen:

Este artículo describe un procedimiento de adaptación de la metodología Métrica 3 a un contexto académico para el desarrollo de trabajos de fin de grado, motivado por una serie de carencias observadas al realizarlos en el Grado en Ingeniería Informática del Software de la Universidad de Oviedo. La metodología de adaptación consistió en la eliminación o simplificación de los procesos de Métrica 3 que se han juzgado menos aplicables al contexto académico y la introducción de elementos y ejemplos que la integran con las asignaturas de la titulación, unificando sus contenidos. Con ello, se ha desarrollado un documento/plantilla que propone una estructura general para un proyecto de desarrollo de software respetando la estructura de Métrica 3. El uso del documento es opcional y flexible: el alumno debe adaptarlo a su trabajo.

La plantilla es pública e integra una serie de estilos y formatos predeterminados, así como explicaciones o ejemplos adicionales que facilitan su uso. El uso de esta plantilla a lo largo de más de 10 años ha sido un éxito, facilitando lograr trabajos con una mejor estructuración. También ha decrementado el número de defectos, problemas generales de desarrollo y dificultades planteadas por los alumnos.

Palabras clave: Desarrollo de software, ingeniero profesional, licenciatura en ingeniería de software, metodología, trabajo de fin de grado

\begin{abstract}
:
This article describes a procedure for adapting the Métrica 3 methodology to an academic context for the development of final degree projects, motivated by a series of deficiencies observed while developing them in the Bachelor of Software Engineering of the University of Oviedo. The adaptation methodology consisted on removing or simplifying of those processes of Métrica 3 that have been judged less applicable to the academic context, and the introduction of elements and examples that integrate it with the degree courses, unifying its contents. Considering this, a document/template has been developed that proposes a general structure for a software development project preserving the structure of Métrica 3 . The use of the document is optional and flexible: the student must adapt it to their work.

The template is public and integrates several default styles and formats, as well as additional explanations or examples that make it easier to use. This template has been successfully used over more than 10 years, facilitating to improve work structure. It also managed to decrease the number of defects, general development problems, and student difficulties.
\end{abstract}

Keywords: Bachelor's degree in software engineering, final degree project, methodology, professional engineer, software development.

\footnotetext{
1 Universidad de Oviedo (España), redondojose@uniovi.es; CÓDIGO ORCID: 0000-0002-0939-0186
} 


\section{INTRODUCCIÓN}

Una de las últimas asignaturas cursadas por los alumnos del Grado en Ingeniería Informática del Software de la Universidad de Oviedo (Informática, 2020) es Dirección y Planificación de Proyectos Informáticos. En ella se aplican los conocimientos y experiencia adquiridos durante el grado para la creación de un proyecto software que, en la medida de lo posible, emula a uno real. Tras esta asignatura se espera que los alumnos sean capaces de transmitir lo que han aprendido al que realmente es su último acto académico: el Trabajo Fin de Grado. Existen varios tipos de trabajos contemplados en nuestra titulación, pero el más frecuente es el de diseño y desarrollo de un producto software que cumpla unas necesidades realistas, dado el objetivo de la titulación.

No obstante, nuestra experiencia pasada es que esta tarea presentaba frecuentemente dificultades cuya resolución no era trivial. Un gran número de alumnos mostraba problemas evidentes al enfrentarse a este trabajo, cuya duración media y complejidad es superior a las actividades de la asignatura mencionada debido a sus restricciones temporales. Entre esos problemas figuran dificultades para abordar el inicio, debido a la gran cantidad de actividades a desarrollar para organizar y empezar a realizarlo, problemas para lidiar adecuadamente con los requisitos/cliente, seleccionar tecnologías, arquitecturas, estructurar las distintas fases del trabajo (cómo se relacionan y secuencian), etc. Todos ellos retrasan el trabajo de los alumnos sobre la planificación esperada $y$, frecuentemente, les hacen incurrir en una pérdida de confianza que perjudica su desarrollo.

Según la experiencia adquirida, de todos los problemas detectados, el más grave es cómo organizar y estructurar toda la información que se genera en las distintas actividades que forman parte de un trabajo de fin de grado. Si bien los alumnos conocen varias formas de hacerlo, su inexperiencia juega en contra de usar de forma efectiva cualquiera de ellas, solicitando muchos de ellos a sus tutores una guía más detallada acerca de cómo proceder en diferentes partes de su realización. Por ese motivo, en la Escuela de Ingeniería Informática de la Universidad de Oviedo se puso en marcha una iniciativa que trata de contrarrestar estas carencias, poniendo un especial cuidado a la hora de organizar y estructurar la información del trabajo. De esta forma, el alumno puede seguir el desarrollo de acuerdo con un orden establecido que le pueda ir guiando.

La iniciativa consiste en proporcionar a los alumnos una plantilla con una estructura bien definida que sigue una metodología de desarrollo de software real, junto con explicaciones que complementan los contenidos de las asignaturas correspondientes a cada parte, ejemplos, materiales de referencia, y todo tipo de ayudas adicionales que permitan reforzar las principales carencias que la experiencia en la dirección de trabajos ha ido identificando con los años. Si bien la plantilla tiene una estructura clara, en todo momento se insiste al alumno en que puede hacerle todas aquellas adaptaciones que considere necesarias, puesto que su proyecto es el que debe dirigir la documentación, y no al revés. El alumno puede añadir o eliminar secciones 0 subsecciones completas si la temática de su proyecto así lo aconsejara.

El resultado de esta experiencia es una mejora en la calidad final de la documentación de los trabajos y una disminución sustancial de las dudas planteadas durante su realización. Los alumnos consiguen así un mayor nivel de seguridad y confianza durante el desarrollo de su trabajo, tener una orientación más clara, y lograr un mayor grado de satisfacción, haciendo más trabajo en menos tiempo. Esto se ha comprobado mediante una encuesta anónima a alumnos que han usado esta plantilla durante los últimos años, que también describiremos al final del artículo. Estos resultados han motivado la mejora continua de esta iniciativa a lo largo de los años y también durante este último curso, a través de un proyecto de innovación docente actualmente en marcha (PINN-19-A-029) (Oviedo, 2019). 
Este artículo describe en detalle cómo se ha creado la plantilla a partir de la metodología, el tipo de documentación que incluye, qué aspectos contempla para mejorar la calidad final de los trabajos, cuál es la opinión de sus usuarios, y otros datos para que esta experiencia pueda reproducirse en otros entornos en los que se juzgue necesaria.

El artículo está dividido así: el capítulo 2 describe por qué se ha seleccionado Métrica 3 entre otras alternativas y detalla paso a paso qué partes de la metodología se han incluido y cuáles no de forma justificada. El capítulo 3 describe aspectos fundamentales de la creación y formato de la plantilla. El capítulo 4 describe el trabajo relacionado mientras que el 5 describe la opinión de alumnos que la han usado. Finalmente, el capítulo 6 detalla las conclusiones y el trabajo futuro.

\section{DESARROLLO DE LA EXPERIENCIA DE INNOVACIÓN}

\subsection{Selección de la metodología}

La decisión más importante para elaborar la plantilla de proyectos de desarrollo es la metodología a seguir. No usar una real no es adecuado debido a que el propósito de un trabajo fin de grado es emular la realización de un proyecto con objetivos realistas, por lo que es adecuado emplear algo cuya experiencia se pueda trasladar directamente al mercado laboral en las circunstancias adecuadas.

No obstante, la decisión de qué metodología usar no es sencilla: existen muchas opciones y en distintas asignaturas del grado se emplean varias. La primera opción estudiada fue el empleo de una ágil, siguiendo el marco de trabajo SCRUM (Takeuchi \& Nonaka, 1986) o el método Kanban (Anderson, 2010). No obstante, se decidió no adoptar como base ninguna de este tipo por no adaptarse a las características del trabajo a desarrollar. Las metodologías ágiles presuponen la existencia de un equipo de profesionales con funciones determinadas que trabajan colaborativamente. También se presupone una estrategia de desarrollo incremental, y entregas periódicas de versiones del producto tras haber finalizado una parte concreta de sus características. Sin embargo, en el contexto académico en el que se desarrolla un trabajo de fin de grado en nuestra escuela el alumno no trabaja en grupo. No existe pues el concepto de equipo de trabajo ni desarrollo colaborativo, al ser una actividad de evaluación fundamentalmente individual.

Por otro lado, el tutor del trabajo rara vez establece una planificación con un conjunto de entregas estricto que el alumno deba seguir, sino que es el alumno el que habitualmente establece el ritmo de trabajo, las partes en las que divide el proyecto, cuáles hace primero y cuánto tiempo estima que pueden Ilevarle hacerlas. El alumno es a la vez jefe de su proyecto, analista y programador, por lo que se vería obligado a emular una serie de conceptos y características de estas metodologías para adaptarlas a un trabajo de fin de grado. Por otro lado, dado que inherentemente favorecen el solapamiento entre diversas fases de desarrollo, su uso puede generar problemas de organización al alumno, al tener que llevar en paralelo diversas actividades.

Por tanto, debido a que el uso de una metodología ágil suponía tener que emular un conjunto grande de elementos, y podría dar algún problema desde el punto de vista organizativo, la plantilla se basó en una metodología con un proceso de desarrollo más lineal y con una organización más procedimental que resulte más fácil de seguir a un alumno: Métrica versión 3 (Portal de Administración Electrónica, 2020) (Cillero, 2020). Esto no quiere decir que un alumno no pueda emplear una metodología ágil para su trabajo si así lo desea: la plantilla siempre es de uso opcional.

Métrica 3 es una metodología de planificación, desarrollo y mantenimiento de sistemas de información del Gobierno de España para la sistematización de actividades del ciclo de vida de los proyectos software de las administraciones públicas. Está basada 
en el modelo de procesos del ciclo de vida de desarrollo ISO/IEC 12207 (ISO, 2008) y la norma ISO/IEC 15504 SPICE (ISO N. , 2020).

Esta metodología está compuesta de una serie de procesos y actividades grande que la hacen compleja. Su aplicación literal a un trabajo de fin de grado es excesiva en la mayoría de los casos. No solo está orientada para un tipo de aplicaciones diferente al que normalmente se va a desarrollar (administración pública en lugar de empresa privada), sino que contempla una serie de aspectos (pasos a producción, planes de mantenimiento, formación de usuarios...) cuya aplicación en un trabajo de fin de grado no se contempla o debería emularse, según su propósito. Por otro lado, la duración estimada de un trabajo de fin de grado en nuestra titulación es de 12 créditos, y es necesario recortar contenidos para adaptarlos al tiempo estimado. La aplicación de la metodología no será por tanto integral, y en la siguiente sección se justificarán los elementos incluidos y excluidos de la plantilla desarrollada en base a la misma.

\subsection{Procesos y actividades de Métrica 3 incluidos}

Métrica 3 está orientada a procesos, y por tanto es necesario que la plantilla los incorpore en el orden y con la nomenclatura establecido por la misma. Cada proceso cuenta con una serie de actividades y dentro de ellas se pueden plantear varias tareas. Es importante destacar que, aunque varios de sus procesos o actividades no han sido incluidos por los motivos que se describirán, el alumno es libre de incluirlos de nuevo si lo estima oportuno y/o de hacerles las modificaciones que necesite para adaptar la metodología a las necesidades particulares de su trabajo. Esto es algo que se insiste en todo el desarrollo del trabajo para que el alumno no vea la plantilla como un marco de trabajo rígido, sino todo lo contrario.

También es importante destacar que los contenidos de la carrera priman sobre los procesos y actividades de la metodología, y a lo largo de la plantilla se insiste mucho en este aspecto: ante una duda acerca de cómo realizar una actividad, se hace referencia a las asignaturas del grado involucradas, y se remarca que siempre sus contenidos primarán sobre lo establecido en la plantilla, en el hipotético caso de que haya algún conflicto para un proyecto determinado. Como se verá más adelante, algunas secciones se han modificado directamente para hacer una adaptación al mundo académico. No obstante, a pesar de estos cambios, la estructura de la metodología original es claramente reconocible en la plantilla.

La Figura 1 detalla las actividades y tareas que se han incluido en la plantilla de los procesos Planificación del Sistema (PSI) y Estudio de viabilidad (EVS) de Métrica 3. 


\section{PLANIFICACIÓN DEL SISTEMA DE INFORMACIÓN (PSI)}

\section{PSI 1: Inicio del Plan de Sistemas de Información}

Análisis de la Necesidad del PSI

Identificación del Alcance del PSI

Determinación de Responsables

PSI 2: Definición y Organización del PSI

Especificación del Ámbito y Alcance

Organización del PSI

PSI 3: Estudio de la Información Relevante

Selección y Análisis de Antecedentes

PSI 7: Definición de la Arquitectura Tecnológica

Identificación de las Necesidades de Infraestructura Tecnológica

Selección de la Arquitectura Tecnológica

\section{ESTUDIO DE VIABILIDAD DEL SISTEMA (EVS)}

\section{EVS 4, 5 y 6: Estudio y Valoración de Alternativas de Solución y Selección de Alternativa Final}

Figura 1. Actividades y tareas de Métrica 3 de los procesos PSI y EVS incluidos en la plantilla. Fuente: Elaboración propia.

En el proceso inicial de Planificación del Sistema de Información (PSI) se han decidido eliminar las actividades PSI 4 (Identificación de Requisitos), PSI 5 (Estudio de Sistemas de Información Actuales), y PSI 6 (Diseño del Modelo de Sistemas de Información), por estar vinculadas a la existencia de un cliente y entorno de implantación real al que hay que hacerles un estudio detallado de sus necesidades prácticamente desde cero, situación que se da muy rara vez en los trabajos de fin de grado.

El estudio e identificación previo de requisitos es imprescindible en un proyecto real. No obstante, se ha decidido usar directamente las actividades homónimas del proceso Análisis del Sistema de Información (ASI) (ver figura 2) en su lugar puesto que, en el contexto académico, las condiciones y requisitos de lo que hay que desarrollar están habitualmente mucho más definidos de partida que en un proyecto real. Si bien a lo largo de las asignaturas afines a la dirección y gestión de proyectos software se insiste en que una adecuada planificación previa, siempre en contacto con el cliente, observando la información manejada, necesidades, instalaciones, etc. es imprescindible, es raro que un trabajo de fin de grado tenga que desarrollarse en circunstancias similares a las de un proyecto de software real para las administraciones públicas para el que Métrica 3 está pensado.

Por un motivo similar se han eliminado las actividades EVS 1 (Establecimiento del Alcance del Sistema), EVS 2 (Estudio de la Situación Actual) y EVS 3 (Definición de Requisitos del Sistema). También se ha fusionado en una sola actividad las EVS 4 (Estudio de Alternativas de Solución), EVS 5 (Valoración de las Alternativas) y EVS 6 (Selección de la Solución). El objetivo es documentar el mismo contenido, pero sin dividirlo en tantas actividades. De esta manera el alumno podrá describir de forma más 
sintética y rápida los límites del sistema a desarrollar, detallar el estado del sistema de partida a mejorar (aunque en la mayoría de los casos partirá de cero), posibles alternativas de solución (si hubiese más de una) y luego decantarse por una de ellas. El objetivo de esta simplificación es eliminar la potencial duplicidad de información con otros apartados, y una de las medidas principales que se tomaron para agilizar el comienzo del trabajo de fin de grado.

\section{ANÁLISIS DEL SISTEMA DE INFORMACIÓN (ASI)}

\section{ASI 1: Definición del Sistema}

Determinación del Alcance del Sistema

\section{ASI 2: Establecimiento de Requisitos}

Obtención de los Requisitos del Sistema

Identificación de Actores del Sistema

Especificación de Casos de Uso

\section{Planificación y Gestión}

Planificación del proyecto

Ejecución del proyecto

Cierre del proyecto

\section{ASI 3: Identificación de Subsistemas de Análisis}

Descripción de los Subsistemas

Descripción de los Interfaces entre Subsistemas

\section{ASI 4: Análisis de los Casos de Uso}

\section{ASI 5: Análisis de Clases}

Diagrama de Clases

Descripción de las Clases

ASI 8: Definición de Interfaces de Usuario

Descripción de la Interfaz

Definición del aspecto de la interfaz

Descripción del Comportamiento de la Interfaz

Diagrama de Navegabilidad

\section{ASI 10: Especificación del Plan de Pruebas}

Figura 2. Actividades y tareas de Métrica 3 del proceso ASI incluidos en la plantilla.

Fuente: Elaboración propia. 
El proceso de Análisis del Sistema de Información (ASI) de Métrica 3 ha sufrido menos recortes que los anteriores (ver Figura 2) al encajar mejor con lo que se espera de un proyecto de desarrollo. Se han eliminado las actividades de Modelo de Datos (ASI 6) y Modelo de Procesos (ASI 7) de cara a poder hacer el análisis más ágil. El producto del ASI 7 se ha integrado como una consecuencia del Análisis de Casos de Uso (ASI 4). ASI 6 (Elaboración del Modelo de Datos) se ha pasado a la fase de diseño cuando, una vez conocidos en detalle los requisitos, se pueden especificar los datos manejados por la aplicación con mayor precisión. De esta manera los datos manejados quedan descritos en un solo lugar de la documentación. La actividad Análisis de Consistencia y Especificación de Requisitos (ASI 9) también se ha eliminado para dar mayor agilidad al desarrollo del proyecto y, a petición expresa del profesorado de la asignatura Dirección y Planificación de Proyectos, se ha incluido un nuevo apartado de "Planificación y Gestión" (que no forma parte de la especificación de Métrica 3) que mimetiza los contenidos que se imparten en dicha asignatura para crear una planificación, calendario de ejecución y cierre del proyecto previos. Este es un ejemplo de que la adaptación que se ha hecho de Métrica 3 no solo consiste en simplificar, sino en adaptar sus contenidos a un contexto académico y, en concreto, de las particularidades de nuestra titulación. Esta nueva sección es también otro motivo por el que se eliminaron los subprocesos del PSI descritos anteriormente.

\section{DISEÑO DEL SISTEMA DE INFORMACIÓN (DSI)}

DSI 3: Diseño de Casos de Uso Reales

DSI 4: Diseño de Clases

Diagrama de Clases

DSI 5: Diseño de la Arquitectura de Módulos del Sistema

Diagramas de Paquetes

Diagramas de Componentes

Diagramas de Despliegue

DSI 6: Diseño Físico de Datos

Descripción del SGBD Usado

Integración del SGBD en Nuestro Sistema

Diagrama E-R

DSI 9: Diseño de la Migración y Carga Inicial de Datos

DSI 10: Especificación Técnica del Plan de Pruebas

Pruebas Unitarias

Pruebas de Integración y del Sistema

Pruebas de Usabilidad y Accesibilidad

Pruebas de Accesibilidad

Pruebas de Rendimiento

Figura 3. Actividades y tareas de Métrica 3 del proceso DSI incluidos en la plantilla. Fuente: Elaboración propia. 
En lo relativo al Diseño del Sistema de Información (DSI), siendo nuestra carrera una ingeniería del software, lo cierto es que el peso de asignaturas que tratan con la arquitectura de los sistemas que soportarán dicho software es bastante limitado dentro del total de créditos impartidos. Por ese motivo, aunque los alumnos tienen los conocimientos básicos acerca de estos temas, se ha decidido eliminar los apartados del diseño más vinculados con la arquitectura en aras a una mayor agilidad. La idea es que se traten en el proceso posterior de implantación, como algo que ya viene prefijado de antemano y que no requiere ser previamente diseñado, puesto que la gran mayoría de trabajos no requieren esta clase de planificación de la arquitectura de soporte tan detallada. Por ese motivo (ver Figura 3) las actividades DSI 1 (Definición de la Arquitectura del Sistema), DSI 2 (Diseño de la Arquitectura de Soporte), DSI 7 (Verificación y Aceptación de la Arquitectura del Sistema) y DSI 8 (Generación de Especificaciones de Construcción) no aparecen en la plantilla, así como también las DSI 11 (Establecimiento de Requisitos de Implantación) y DSI 12 (Aprobación del Diseño del Sistema de Información).

La Construcción del Sistema de Información (CSI) es otra de las partes de la metodología que más ha conservado su estructura en la plantilla (ver Figura 4), habiéndose eliminado únicamente la Definición de la Formación de los Usuarios Finales (CSI 7), por no ser aplicable en nuestro contexto, y la Aprobación del Sistema de Información (CSI 9), que es realmente una tarea más propia del tribunal que evalúe el trabajo. En esta parte se espera que el alumno ponga de manifiesto todas las habilidades, técnicas y conceptos para el desarrollo de software robusto y de calidad que ha aprendido durante sus estudios (Ortin, Redondo, \& Quiroga, 2017) (Redondo, 2019a) (Redondo, 2019b) (Redondo, 2019c).

Otra de las partes más recortadas de la metodología corresponde al proceso de Implantación y Aceptación del Sistema (IAS) y al Mantenimiento de Sistemas de Información (MSI). Del primer proceso se han eliminado la Formación Necesaria Para la Implantación (IAS 2), Incorporación del Sistema al Entorno de Operación (IAS 3), Pruebas de Aceptación del Sistema (IAS 6) y el Establecimiento del Acuerdo de Nivel de Servicio (IAS 8). Además, se ha unificado en una sola actividad la Presentación y Aprobación del Sistema y el Paso a Producción (IAS 9 e IAS 10). Debe tenerse en cuenta que, incluso aunque el trabajo sea para un cliente real, muy rara vez el proyecto se presenta con una puesta en producción completa por falta de tiempo, y tampoco es un requisito que se exija en estos casos.

Finalmente, también se ha eliminado completamente el proceso Mantenimiento de Sistemas de Información (MSI), puesto que, de hacerse, tendría que ser ya con el proyecto en manos de un potencial cliente real, y como parte de la vida laboral del proyectante. No obstante, todo esto es susceptible a cambio y adaptación a las particularidades de cada proyecto concreto. Particularmente, de vez en cuando algún proyecto presentado sí se encuentra desplegado y en producción, puesto que se derivó del trabajo que el alumno desarrolla realmente en su empresa. Este apartado sí aparece entonces para reflejar las labores de mantenimiento que el alumno realiza en su empresa periódicamente.

Ya fuera de metodología Métrica 3 se incluyen unos Apéndices más relacionados con el ámbito académico:

- Problemas Encontrados Durante el Desarrollo: donde el alumno puede detallar los problemas más relevantes que ha encontrado durante el desarrollo,

- Plan de gestión de riesgos: nuevamente a petición de la asignatura de Dirección y Planificación de Proyectos Informáticos

- Conclusiones

- Ampliaciones: trabajo futuro, normalmente sólo planificado 
- Referencias bibliográficas: sique el formato estandarizado IEEE para que los alumnos citen adecuadamente sus fuentes.

\section{CONSTRUCCIÓN DEL SISTEMA DE INFORMACIÓN (CSI)}

\section{CSI 1: Preparación del Entorno de Generación y Construcción}

Estándares y normas seguidos

Lenguajes de programación

Herramientas y programas usados para el desarrollo

CSI 2: Generación del Código de los Componentes y Procedimientos

CSI 3: Ejecución de las Pruebas Unitarias

CSI 4: Ejecución de las Pruebas de Integración

\section{CSI 5: Ejecución de las Pruebas del Sistema}

Prueba de Usabilidad

Pruebas de Accesibilidad

CSI 6: Elaboración de los Manuales de Usuario

Manual de Instalación

Manual de Ejecución

Manual de Usuario

Manual del Programador

CSI 8: Construcción de los Componentes y Procedimientos de Migración y Carga Inicial de Datos

\section{IMPLANTACIÓN Y ACEPTACIÓN DEL SISTEMA (IAS)}

IAS 1: Establecimiento del Plan de Implantación

IAS 4: Carga de Datos al Entorno de Operación

IAS 5: Pruebas de Implantación del Sistema

IAS 7: Preparación del Mantenimiento del Sistema

IAS 8: Establecimiento del Acuerdo de Nivel de Servicio

IAS 9-10: Presentación y Aprobación del Sistema y Paso a Producción

Figura 4. Actividades y tareas de Métrica 3 de los procesos CSI e IAS incluidos en la plantilla. Fuente: Elaboración propia.

\subsection{Interfaces de Métrica 3}

Métrica 3 proporciona cuatro interfaces que definen actividades orientadas a la mejora y perfeccionamiento de sus procesos principales para garantizar que se consigan los objetivos de desarrollo. Estos interfaces se integran en la realización del trabajo, pero no aparecen de forma explícita como tales en la memoria. Esta decisión se ha tomado para simplificar la documentación y ejecución del proyecto, y para integrar mejor las asignaturas correspondientes de la carrera. 
De esta manera, las interfaces de Gestión de proyectos (GP) y Aseguramiento de la Calidad (CAL) se contemplan en los apartados añadidos mencionados correspondientes a la asignatura de Dirección y Planificación de Proyectos Informáticos. El apartado de Seguridad (SEG) se contempla en las explicaciones y ejemplos de la plantilla, pero dirigiendo siempre a los contenidos relacionados de la asignatura Seguridad de Sistemas Informáticos (Redondo, 2019d) (Redondo, 2019e), que lidia con diversos aspectos relativos a la creación de software más seguro y que los alumnos ya están acostumbrados a incluir como parte de la documentación de las diferentes partes de su software. Finalmente, la Gestión de la Configuración (GC), debido a que nuestra carrera no se especializa en este apartado, queda integrada también en las explicaciones y ejemplos de la plantilla, vinculándola con la asignatura de Administración de Sistemas y Redes y proporcionándose también enlaces a otros materiales que pueden ser útiles (Redondo, 2019f).

\subsection{Técnicas de desarrollo, diagramas y perfiles}

De las técnicas de desarrollo y diagramas especificados por Métrica 3, la plantilla menciona expresamente los siguientes, sin excluir cualquier otro elemento que pueda adaptarse mejor a las necesidades del alumno o el proyecto.

- Casos de uso

- Diagramas de clases

- Diagrama de componentes

- Diagrama de despliegue

- Diagrama de flujo de datos

- Diagrama de interacción

- Diagrama de secuencia

- Diagrama de colaboración

- Diagrama de paquetes

- Diagrama de transición de estados

- Modelo entidad/relación extendido

- Técnicas de gestión de proyectos adaptadas a la asignatura correspondiente

- Planificación
○ PERT
- Diagrama de Gantt
- Estructura de descomposición del trabajo

Finalmente, la plantilla está pensada para que el alumno desarrolle las labores de los siguientes perfiles contemplados en Métrica: Jefe de Proyecto, Consultor, Analista y Programador del trabajo.

\section{CREACIÓN DE LA PLANTILLA}

Uno de los primeros aspectos en los que se ha tenido especial cuidado a la hora de crear la plantilla es la creación de un conjunto de estilos y formato unificado para el documento. Esto puede parecer un aspecto superficial, pero nuestra experiencia previa demostró que existían problemas importantes en este sentido. La mayor parte de los alumnos perdían mucho tiempo lidiando con aspectos propios del programa de edición (la mayoría, Microsoft Word). Además, los documentos presentados carecían de elementos oficiales que les identificaran como trabajos de fin de grado de la Universidad de Oviedo, incluso aunque la inclusión de estos estuviese reglada. Esto hacía que muchos proyectos incurriesen en defectos de forma que podrían afectar a su calificación. 
En cuanto a formato, además de proporcionar una galería de estilos fija con el documento (texto normal, imágenes, títulos de diferentes niveles, pies de imagen y tabla...) y ejemplos de formato para elementos comunes (tablas, listas de puntos...), se incluyó una cabecera de página oficial obligatoria (ver Figura 5).

Otro de los problemas existentes es que, aunque los contenidos de la portada del trabajo de fin de grado están prefijados, era frecuente encontrarse con trabajos que carecían de alguno de ellos. Por ese motivo, la plantilla proporciona directamente todos los elementos comunes de la portada, y el alumno solo tiene que rellenar sus datos personales y título (ver figura 6). Todas estas acciones de cara a unificar el formato permiten que los proyectos tengan una apariencia oficial, coherente y usando colores, escudos y elementos de acuerdo con las normativas de la Universidad de Oviedo, evitando defectos de forma y pérdidas de tiempo debido a cuestiones meramente de estilo y formato, o problemas de edición. También se proporcionan elementos para automatizar la creación de índices de contenidos, de ilustraciones y referencias bibliográficas.
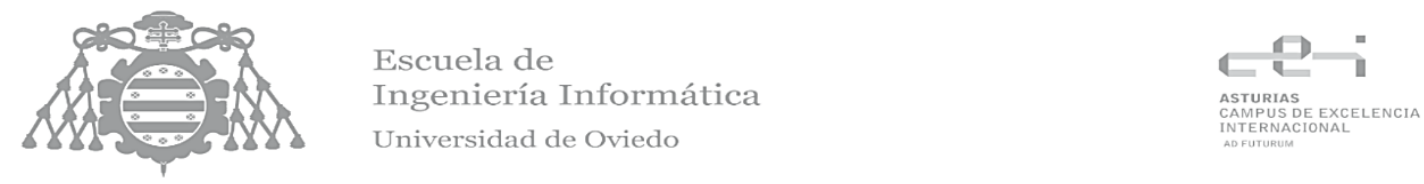

Figura 5. Cabecera oficial de páginas obligatoria.

Fuente: Elaboración propia.

Finalmente, debido a que la plantilla se actualiza frecuentemente de cara a responder a cambios en la titulación y de los contenidos de las asignaturas vinculadas con ella, se incorpora al principio un historial de cambios entre versiones. Esta información permite a un alumno que esté usando una versión de la plantilla actualizarla fácilmente, al tener un lugar donde consultar las modificaciones que se han hecho detalladamente y poder así incorporarlas a su versión actual. Finalmente, la plantilla incorpora algunas instrucciones de uso y el acuerdo de licencia completo GNU Free Documentation License (Foundation, 2020) bajo el que publica, al ser un requisito de este tipo de licencia. La plantilla se suministra en formato Word 2007 o superior y Latex, si bien en estos momentos solo la primera versión se está actualizando.

Uno de los objetivos de la plantilla es integrar todos los contenidos relevantes impartidos en la titulación en un solo documento para que se usen conjuntamente de cara a desarrollar el trabajo de fin de grado. No obstante, una sola persona no puede hacer este trabajo por si sola, y es necesaria la colaboración de gran parte del profesorado. En este sentido se han hecho dos esfuerzos:

- Mapeo de las secciones de la plantilla con las distintas asignaturas de la titulación. A través de las guías docentes públicas de las asignaturas de la titulación, y de los conocimientos propios del autor, se hizo un mapa que vincula cada sección de la plantilla con las potenciales asignaturas que imparten contenidos relacionados con las mismas, trasladándose dicha relación a los textos de ejemplo de la plantilla de forma explícita.

- Consulta con los expertos en las materias. Tras el mapeo, se contactó al profesorado de dichas asignaturas para recabar su opinión acerca de los ejemplos, tutoriales o guías relacionadas que se podrían incorporar, y aportar cualquier otra clase de información que ayude a los alumnos a usar los contenidos de las asignaturas de forma efectiva en un trabajo de fin de grado. $\mathrm{Ha}$ de tenerse en cuenta que los alumnos podrían haber cursado algunas asignaturas hace mucho tiempo, y por tanto estos ejemplos son de mucha ayuda 
para recordar o actualizar sus contenidos. Por otro lado, se especifica claramente que todo ejemplo proporcionado se hace con ánimo de resolver dudas y servir de guía, pero que nunca es un sustituto de los contenidos de las asignaturas correspondientes.

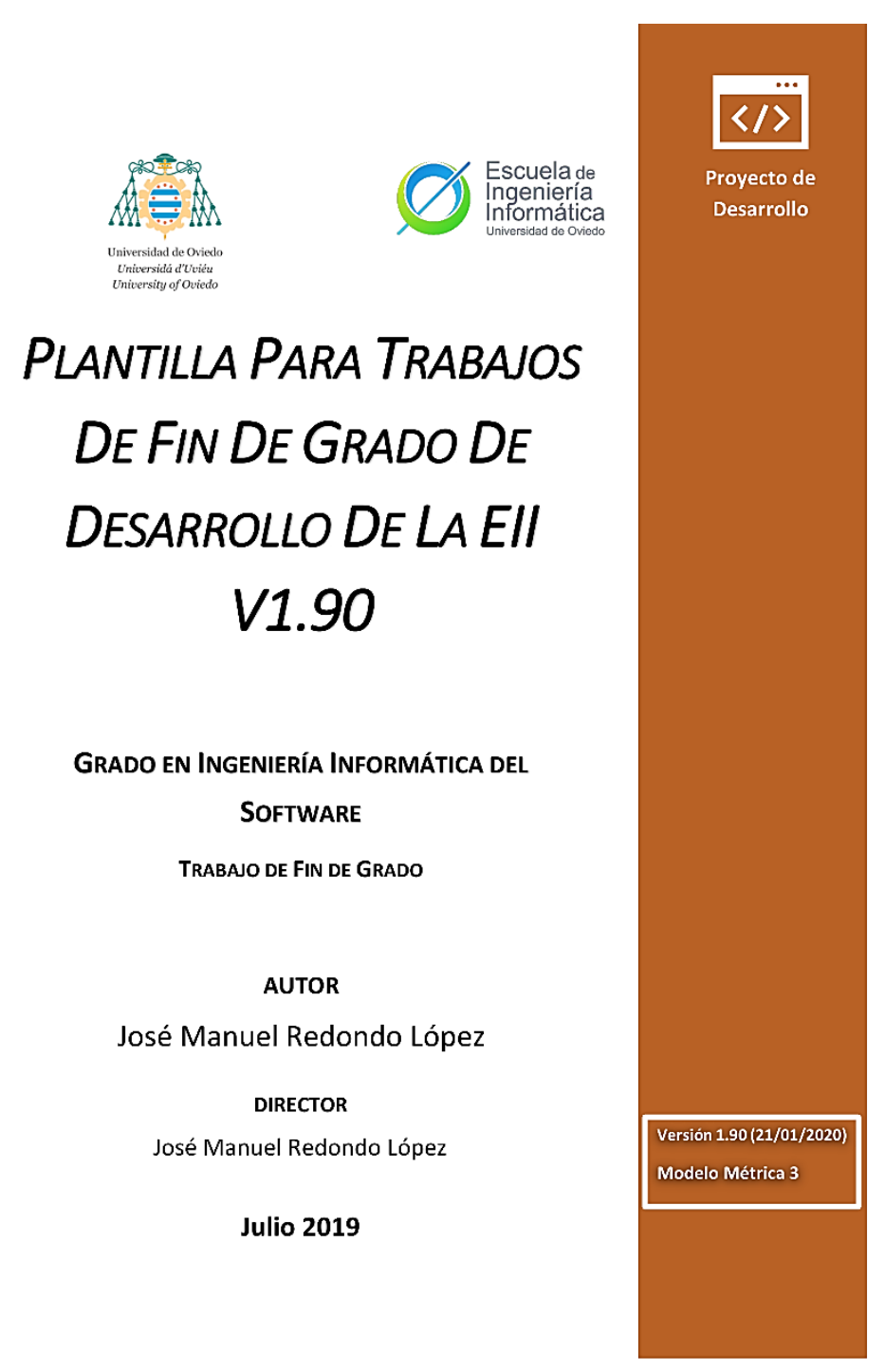

Figura 6. Portada oficial de los trabajos de fin de grado.

Fuente: Elaboración propia.

La figura 7 muestra una de las explicaciones que la plantilla da a sus usuarios. Estas explicaciones no se limitan a textos cortos, sino que también pueden incorporar ejemplos proporcionados por el autor o por los expertos en las distintas materias consultados. Si la sección está vinculada a una o varias asignaturas, el nombre de estas se destaca en rojo para que el alumno sepa a simple vista de qué asignaturas debe repasar contenidos de forma prioritaria para completar la sección correspondiente. En la figura 7 también se aprecia que los textos de ejemplo se muestran con un estilo independiente (otro color, cursiva) para distinguirlos del texto normal y poder eliminarlos más fácilmente cuando ya no sean necesarios. Esto se ha hecho en una de las últimas revisiones de la plantilla realizadas, debido a que se han detectado casos de trabajos que han dejado alguno de estos textos en su versión final por error. 


\section{ASI 2: ESTABLECIMIENTO DE REQUISITOS}

En adelante será necesario hacer determinados diagramas en varias secciones del documento. Además de Visio o la herramienta Linux Dia, los siguientes enlaces contienen herramientas que podrian ser útiles para hacer diagramas propios de dichas secciones:

- https://www.planttext.com/

- https://yuml.me/diagram/nofunky/usecase/draw

- https://www.websequencediagrams.com/

- https://www.quickdatabasediagrams.com/

- https://www.lucidchart.com/pages/es

\section{Obtención de los Requisitos del Sistema}

El producto de esta sección se crea para su aprobación formal, es decir, que los potenciales clientes deben ver a partir de él las especificaciones completas del sistema. Además, esta sección construirá una base para solicitar cambios en los requisitos antes de avanzar más en la construcción del sistema. En esta sección tiene prioridad siempre todo lo aprendido en la asignatura "Ingeniería de Requisitos" de tercer curso, cuyos conocimientos deben aplicarse aquí.

Los requisitos del sistema se deben mostrar en una tabla como la que se presentará a continuación con ejemplos, ordenados por algún criterio lógico en función de a lo que se refieran. Por ejemplo, si tenemos usuarios tiene sentido agrupar todos los requisitos que tengan que ver con los usuarios.

Tampoco es necesario crear una única tabla para todos los requisitos, pueden crearse varias tablas que agrupen los requisitos que se refieran a una entidad. En general se recomienda hacerlo como se haya explicado en la asignatura mencionada.

Este apartado debe incluir también antes de la tabla de requisitos, si existe como tal, la especificación textual que el cliente nos proporcione sobre la aplicación, fruto de las reuniones que hayamos tenido con él o de las entrevistas que podamos haber llevado a cabo. Ha de tenerse en cuenta que esta información es la que usaremos para extraer los requisitos de la aplicación, por lo que no debe faltar sin contamos con ella.

\begin{tabular}{|l|ll|}
\hline CÓDIGO & NOMBRE REQUISITO & DESCRIPCIÓN DEL REQUISITO \\
\hline R1.1 & Insertar Usuario & $\begin{array}{l}\text { Se debe añadir un usuario al sistema una vez leídos y } \\
\text { validados sus datos. }\end{array}$ \\
$R 1.2$ & Leer Datos Usuario & $\begin{array}{l}\text { Deben pedirse los datos completos de un usuario del sistema } \\
\text { R1.2 }\end{array}$
\end{tabular}

Plantilla para Trabajos De Fin De Grado De Desarrollo De la EII V1.90

Figura 7. Ejemplo de las explicaciones que la plantilla incorpora a sus secciones.

Fuente: Elaboración propia.

\section{RESULTADOS}

Esta iniciativa comenzó en 2008 con una primera versión, que fue evolucionando a medida que las tecnologías y las asignaturas asociadas también lo hacían. Por ello, la plantilla ha tenido mejoras y adaptaciones continuas para integrarla mejor con los contenidos de la carrera, estando las últimas en marcha durante 2020. No obstante, estos cambios han sido fundamentalmente a los ejemplos y a los contenidos de los 
apartados; la estructura base de la plantilla no ha cambiado, dado que Métrica 3 no ha sufrido evoluciones reseñables desde entonces.

Siendo una iniciativa de tan largo recorrido, ha sido usada por un gran número de alumnos para la finalización de sus estudios. Aunque en principio se pensó únicamente para facilitar el trabajo a los alumnos tutorizados por el autor, la idea gustó mucho a otros compañeros que también la usaron de la misma manera, aumentando así el número de potenciales usuarios.

Para evaluar la utilidad que ha tenido esta plantilla a lo largo de todos estos años, se ha creado una encuesta muy breve en la que exalumnos o alumnos en proceso de finalización contestaban fundamentalmente dos aspectos: si la plantilla les había sido útil o no y qué tipo de uso le habían dado. Esta información se ha recogido de forma no anónima, pidiendo además el título del proyecto y el año de lectura para verificarla contra la registrada en la Biblioteca de Proyectos de la Universidad de Oviedo. Para ello, se ha creado un cuestionario vía Microsoft Forms en el que previamente el alumno debe validarse con su cuenta de usuario personal de la universidad para realizarlo. Para aquellos casos que no conserven su cuenta, se ha habilitado un proceso de recogida de respuestas por correo electrónico, verificando manualmente la identidad del remitente. La encuesta fue respondida por 81 alumnos, cuyos proyectos fueron terminados desde el año 2008 a la actualidad. Dichos alumnos no son solo tutorizados por el autor, sino que muchos de ellos lo fueron por otros profesores. Es muy probable que el número total de alumnos que han usado esta plantilla sea sustancialmente superior, dado que su uso es muy popular (la escuela recomienda el uso de la plantilla, pero siempre de forma opcional), pero se trata de un volumen de respuestas suficiente para que los datos obtenidos tengan representatividad.

La figura 8 resume la forma de uso de la plantilla declarada por los alumnos. En ella se puede ver que prácticamente 3 de cada 4 alumnos dice haber usado su estructura en su mayor parte tal cual se ha descrito, haciendo adaptaciones menores. Para el resto, algunas de las secciones de la plantilla le fueron útiles para completar su documentación, pero la estructura completa en sí no fue usada. Esto indica que el modelo de plantilla planteado se adapta muy bien a una gran cantidad de los trabajos que se hacen en la escuela, por lo que las decisiones tomadas en cuanto a su planteamiento y estructura parecen adecuadas. El resto de los trabajos han sacado provecho de la idea de forma sustancial, puesto que nadie declara haber usado la plantilla para simplemente sacar información de importancia menor o poco significativa.

La utilidad de la plantilla percibida por los alumnos es aún más positiva. La figura 9 muestra que el 95\% de los usuarios que hicieron la encuesta encuentran la iniciativa muy útil, un $5 \%$ creen que les ha ayudado a ahorrar tiempo, y ninguno declara que la plantilla no resultó ser lo que esperaba de cara a afrontar el trabajo fin de grado. Todo ello nos indica que la aproximación seguida ha sido la adecuada y que realmente está ayudando a los alumnos, adaptándose muy bien a las lógicas variantes que cada proyecto pueda tener respecto a otros. 


\section{Forma de Uso de la Plantilla}

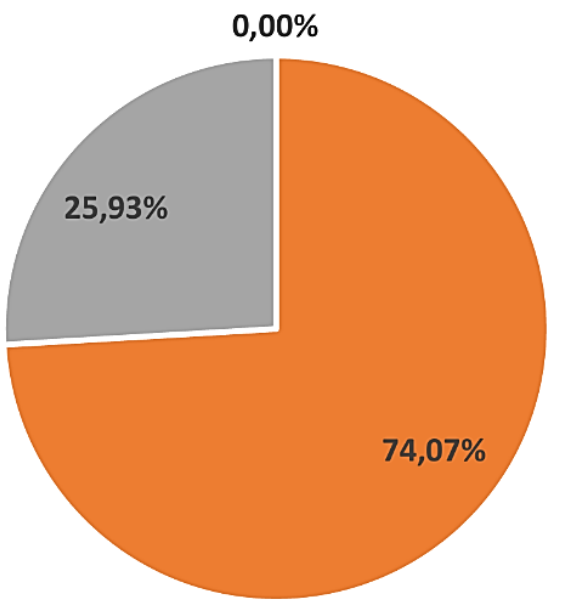

- Basé todo el proyecto en su estructura, aunque hiciese algunas adaptaciones

घ Usé algunas secciones completas, pero no toda la estructura

॥ Solo usé algunas ideas / apartados

Figura 8. Forma de uso de la plantilla.

Fuente: Elaboración propia.

¿Te ha resultado útil?

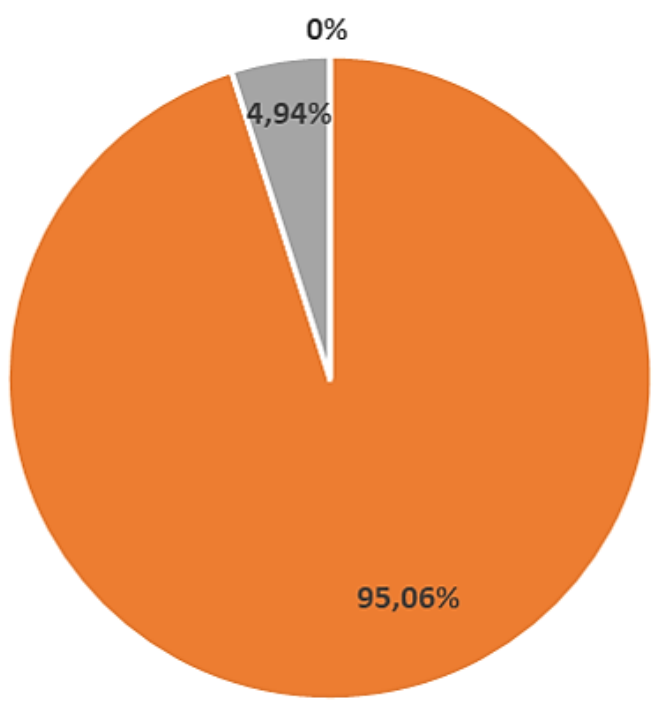

घ Sí, mucho $=$ Me ayudó a ahorrar algo de tiempo $\quad$ No, no era lo que me esperaba

Figura 9. Utilidad de la plantilla para los alumnos.

Fuente: Elaboración propia.

Por otro lado, algunos compañeros han destacado que gracias a esta iniciativa se han eliminado muchos defectos de forma y se han eliminado prácticamente los casos en los que un trabajo de fin de grado presentaba alguno de sus apartados "mayores" de 
forma completamente inadecuada o muy escasa, aumentando la calidad y calificación media de los mismos. El número de trabajos de fin de grado tutorizados por el autor que han seguido esta plantilla en la actualidad está cercano a 70, con una calificación media de $9,4 / 10$ puntos.

Finalmente, algunos alumnos o personas ajenas a la institución han comunicado al autor otros usos adicionales de la plantilla. Por ejemplo, se sabe que esta plantilla ha sido usada, de forma adaptada, en otras universidades españolas a petición de alumnos que estaban buscando una solución similar para su trabajo de fin de grado. También se tiene constancia de su uso adaptado en otras titulaciones de ingeniería de la Universidad de Oviedo.

\section{TRABAJO RELACIONADO}

El uso de plantillas para facilitar la creación de trabajos de fin de grado o máster en titulaciones de ingeniería es común en la universidad española, si bien normalmente dichas plantillas no detallan tanto su contenido como la descrita en este artículo, limitándose a ser una guía de estilos al estilo de lo que se ofrece en muchas revistas, plantear una estructura abstracta que no sigue ninguna metodología real, o bien ser simplemente un compendio de distintas secciones obligatorias y sus portadas en formato oficial, que luego el alumno debe integrar en su trabajo final. Esto deja al alumno un grado de libertad muy grande sobre los contenidos de sus trabajos, que en nuestra experiencia es lo que lleva a los problemas que se tratan de solventar con la plantilla que hemos descrito en este artículo.

Un primer ejemplo lo tenemos en la Universidad de Extremadura (Cáceres, 2020) cuya plantilla incluye un formato y estilos oficiales unificados para todos los trabajos, pero plantea solo una estructura básica para los mismos en $5-7$ puntos (sin incluir referencias y anexos), sin textos explicativos de ninguna clase (no sigue ninguna metodología), e incluyendo todos los modelos de proyectos admitidos en un solo documento. Se ofrecen versiones Word y Latex.

Una aproximación similar a la anterior la podemos encontrar en la Universidad de Valladolid (Ramirez Osarno, 2020), donde la plantilla ofrecida también es una guía de estilos y formato oficial, y está compuesta por una guía de unos pocos puntos que contemplan una estructura general sin seguir una metodología concreta. No obstante, en este caso si se proporcionan, como documentos individuales, otras partes del trabajo que se pueden incluir en función del carácter de este (presupuesto, planos, anejos...), así como la portada y las separatas entre las distintas secciones, dando algo más de soporte al alumno en cuestión de estructura y formato de su trabajo. En este caso, solo se ofrecen documentos en formato Word (.doc). Otro ejemplo podemos encontrarlo en la Escuela Politécnica de Gijón (Gijón, 2020) donde, además de formato y estructura general en la misma línea que la plantilla anterior, se mezclan en un solo documento, sólo en formato Word, la normativa de tramitación de los proyectos e información para todos los tipos de proyectos que esta escuela admite. Anexos como presupuestos, planos, etc. están descritos en el propio documento, pero la portada oficial se ofrece como descarga separada.

Un tercer ejemplo de las plantillas que ofrecen una estructura general, pero que no siguen ninguna metodología realista, lo podemos ver en la Universidad Carlos III de Madrid (UC3M, 2020), donde la plantilla ofrecida contempla también formatos Word y Latex e incluye de nuevo una guía de unos pocos puntos que marcan una estructura abstracta del proyecto a seguir. No obstante, en este caso si que se ofrece algo más de detalle para guiar al alumno, especialmente en los primeros apartados del trabajo (introducción, estado del arte y análisis de la situación), donde se pueden encontrar menciones explícitas a incluir una motivación del trabajo, objetivos, un marco regulador, 
hacer una descripción de la situación actual y posibles soluciones a los problemas observados. Finalmente, también se ofrece una estructura general para los requisitos de la solución a crear para facilitar al alumno el comienzo de su trabajo fin de grado, lo que está alineado con los objetivos de nuestro trabajo. No obstante, la guía proporcionada finaliza aquí y el resto del trabajo ya no está tan detallado hasta llegar a las conclusiones, donde sí se hace mención explícita a describir si se han cumplido los objetivos y a incluir un trabajo futuro. No se proporciona ninguna explicación o ejemplo más allá de formatos.

De entre las plantillas que ofrecen ejemplos más cercanos a lo que habitualmente se ofrecen como guía de publicación en muchas revistas, podemos encontrar en la Universidad Politécnica de Valencia (Valéncia, 2020) que ofrece, en formato Word y Latex, una plantilla que se limita a especificar el formato de elementos como tablas, párrafos, figuras, etc. pero sin dar ninguna guía respecto a sus contenidos más allá de especificar un resumen inicial. De manera similar, la Universidad de la Laguna (Colebrook, 2020) ofrece, solo en formato Word, prácticamente los mismos elementos, si bien indica expresamente la presencia de apartados como introducción, conclusiones, presupuesto y apéndice, pero de nuevo sin entrar en el contenido del trabajo de fin de grado. Cabe destacar que esta plantilla usa una licencia Creative Commons de un carácter similar a la que se usa en la plantilla descrita en este artículo.

Finalmente, el uso de plantillas para trabajos de fin de grado también se encuentra en otros ámbitos fuera de la ingeniería, si bien tienen un carácter similar a los ya descritos. Un ejemplo lo podemos ver en el grado de Ciencias del Deporte de la Universidad de Murcia (Abraldes Valeiras, 2020) donde, en lugar de una estructura a seguir en el proyecto, se entrega una especie de manual de uso de Word que describe todos aquellos elementos del editor que pueden resultar útiles para desarrollar el mismo, así como los criterios de valoración que el tribunal usará para valorar el proyecto, lo cual es un añadido muy interesante de cara a que el alumno sepa exactamente con qué se va a encontrar en la defensa del mismo.

\section{CONCLUSIONES}

Dado el éxito de la plantilla y sus contenidos declarado por sus usuarios, se considera que el objetivo de su creación se ha logrado de forma adecuada y que, por tanto, es una herramienta capaz de ayudar a la realización de trabajos de fin de grado. Con esta plantilla los alumnos logran tener una guía adecuada de cómo desarrollar su trabajo, con una estructura clara y adaptable que garantiza que no se omita ninguna sección importante por error. Al mismo tiempo, es flexible para adaptarse a distintas necesidades, enlaza con distintas asignaturas de la titulación para completar sus contenidos, proporciona facilidades para usar un formato unificado a la hora de presentar los trabajos (evitando defectos de forma) y es una forma de ahorrar tiempo de documentación y desarrollo al disminuir el número de dudas planteadas por los alumnos. Debido al éxito de la iniciativa, también se mantiene en paralelo una versión de la plantilla descrita para el Máster en Ingeniería Web que se imparte en nuestra escuela (Escuela de Ingeniería Informática, 2020), de idéntico contenido, pero con ejemplos vinculados a las asignaturas concretas de esta titulación. El autor ha tutorizado unos 20 trabajos de este máster que la han usado, con una nota media de 9,3/10 puntos. Finalmente, la plantilla que la escuela desarrolló para otro tipo de trabajos de fin de grado aceptados, los Proyectos de Experiencia Profesional, usaba los estilos y formatos descritos para esta plantilla (los contenidos eran necesariamente diferentes debido al carácter de estos trabajos).

Como trabajo futuro se desarrollará una variante con contenidos totalmente diferentes adaptada a proyectos de investigación del citado máster. También se harán algunos cambios a ciertas secciones fruto de colaboraciones con otros expertos de las 
asignaturas del grado para mejorar los ejemplos. Por último, se plantea evolucionar la versión actual en Latex para que sus contenidos sean idénticos a las de formato Word, ya que parece que la demanda de alumnos de un documento base en este formato está aumentando.

Dada su licencia, y potencial utilidad en otras carreras vinculadas a la ingeniería del software, la plantilla, en formato editable, se encuentra disponible públicamente de forma gratuita (Redondo, 2020).

\section{AGRADECIMIENTOS}

Este trabajo ha sido parcialmente financiado por el Ministerio de Ciencia, Innovación y Universidades, proyecto RTI2018-099235-B-I00. También ha sido parcialmente financiado por el proyecto GR-2011-0040 de la Universidad de Oviedo. En la actualidad su desarrollo, actualización y mejora forma parte del proyecto de innovación docente PINN-19-A-029 de la Universidad de Oviedo.

\section{REFERENCIAS}

Abraldes Valeiras, J. A. (22 de 5 de 2020). Modelo de documento del TFG. Guía para el alumno. Recuperado https://www.um.es/documents/378246/12599300/INSTRUCCIONES.+2019-03-

06+Modelo+TFG+v1.0.pdf/e4d39c7d-2541-4302-a10a-354a56303edc

Anderson, D. J. (2010). Kanban: Successful Evolutionary Change for Your Technology Business. Blue Hole Press.

Cáceres, E. P. (22 de 5 de 2020). Trabajo Fin de Grado. Plantillas. Recuperado de https://www.unex.es/conoce-la-uex/centros/epcc/informacion-academica/tfestudios/tfeg/plantillas

Cillero, M. (2020). MÉTRICA 3. Recuperado de https://manuel.cillero.es/doc/metrica-3/

Colebrook, M. (22 de 5 de 2020). Plantillas. Recuperado de https://github.com/mcolebrook/Plantillas/blob/master/Plantilla_TFG_v2.0.pdf

Escuela de Ingeniería Informática. (30 de 4 de 2020). Máster de Ingeniería Web. Recuperado de https://miw.uniovi.es/

Foundation, G. (30 de 4 de 2020). GNU Free Documentation License. Recuperado de https://www.gnu.org/licenses/fdl-1.3.html

Gijón, E. (22 de 5 de 2020). Trabajo Fin de Grado. Recuperado de http://epigijon.uniovi.es/estudiantes/tfg

Informática, E. d. (2020). Grado en Ingeniería Informática del Software. (Universidad de Oviedo) Recuperado http://ingenieriainformatica.uniovi.es/infoacademica/grado

ISO. (2008). ISO/IEC 12207:2008 Systems and software engineering - Software life cycle processes. Recuperado de https://www.iso.org/standard/43447.html

ISO, N. (2020). El Estándar internacional ISO/IEC 15504. Recuperado de https://www.normas-iso.com/iso-iec-15504-spice/

Ortin, F., Redondo, J., \& J.Quiroga. (2017). Design and evaluation of an alternative programming paradigms course. Telematics. and Informatics, 34(6), 813-823.

Oviedo, U. d. (2019). Proyectos de Innovación Docente 2019. (Universidad de Oviedo) Recuperado de http://www.innova.uniovi.es/innovaciondocente/pinn/2019 
Portal de Administración Electrónica. (2020). Métrica v.3. (Gobierno de España) Recuperado de https://administracionelectronica.gob.es/pae_Home/pae_Documentacion/pae_ Metodolog/pae_Metrica_v3.html

Ramirez Osarno, P. (22 de 5 de 2020). Plantillas - Trabajos Fin de Carrera. Recuperado de http://etsiiaa.uva.es/?p=1063

Redondo, J. (2019a). New features of C\# 5 and 6 . Recuperado de www.researchgate.net/publication/330223681_New_Features_of_C_5_a nd_6

Redondo, J. (2019b). New features of C\# 7.X. Recuperado de https://www.researchgate.net/publication/330358763_New_Features_of_CShar p_7X

Redondo, J. (2019c). New features of C\# 8 and beyond. Recuperado de www.researchgate.net/publication/330514620_New_Features _of_CSharp_8_and_beyond

Redondo, J. (2019d). Are You Breath-hacking? Introducción (en Español) al mundo del hacking con un ejemplo práctico. Recuperado el 25 de 10 de 2019, de https://www.researchgate.net/publication/334303691_Are_You_Breathhacking_Introduccion_en_Espanol_al_mundo_del_hacking_con_un_ejemplo_p ractico

Redondo, J. (2019e). Seguridad Web Independiente del Lenguaje. doi:10.13140/RG.2.2.12921.01127/1

Redondo, J. (2019f). Introducción Práctica a la Administración Segura de Servidores Apache Bajo Linux. Oviedo. Oviedo (Asturias): Servicio Publicaciones Universidad de Oviedo.

Redondo, J. M. (30 de 4 de 2020). Documentos-modelo para Trabajos de Fin de Grado/Master de la Escuela de Informática de Oviedo. Recuperado de https://www.researchgate.net/publication/327882831_Documentos-

modelo_para_Trabajos_de_Fin_de_GradoMaster_de_la_Escuela_de_Informati ca_de_Oviedo

Takeuchi, H., \& Nonaka, I. (1986). The New New Product Development Game. Harvard Bussiness Review.

UC3M. (22 de 5 de 2020). Trabajo de Fin de Grado UC3M: Escribir el TFG. Recuperado de http://uc3m.libguides.com/TFG/escribir

Valéncia, U. P. (22 de 5 de 2020). Documentos y Plantillas. Recuperado de http://www.upv.es/titulaciones/GITTEL/info/1054178normalc.html 